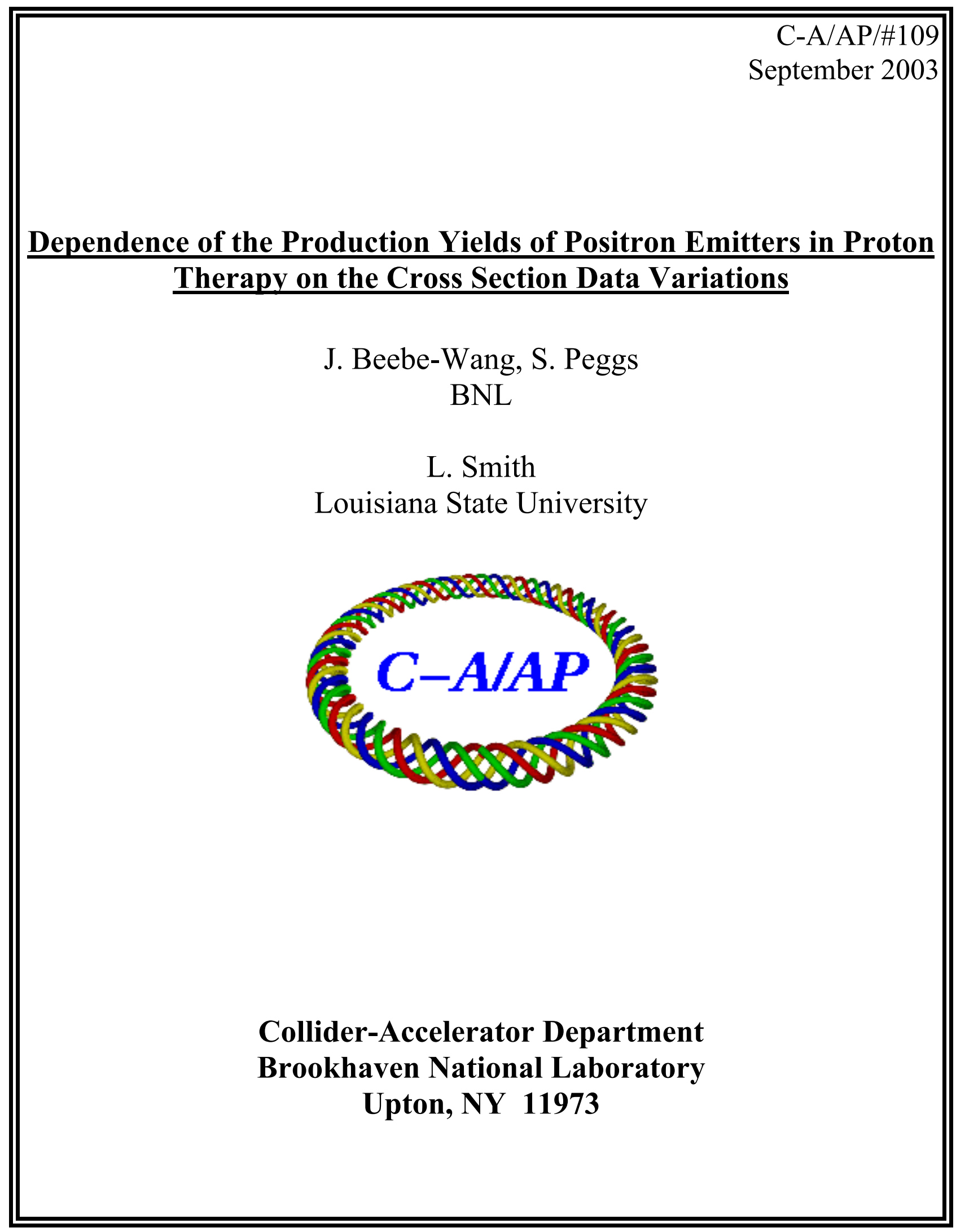




\title{
Dependence of the Production Yields of Positron Emitters in Proton Therapy on the Cross Section Data Variations
}

\author{
Joanne Beebe-Wang, Stephen Peggs \\ Collider Accelerator Department, Brookhaven National Laboratory \\ Lindsay Smith \\ Louisiana State University
}

\begin{abstract}
Earlier works, including a recent one at BNL, demonstrated that positron emission tomography (PET) is a potentially powerful tool for quality assurance and the treatment planning of Proton Therapy (PT). In particular, the PET images show the adequacy of the overlapping of the dose distribution with the intended target volume. Here, we present calculations of the possible variations in yields of positron emitters produced by proton beams of $250 \mathrm{MeV}$ in a soft tissue, and combine the results with the Monte Carlo simulations of PET data collection from our recent work to show the effect of input data variations on the images. We demonstrate that the image results depend strongly on the available nuclear reaction cross section data.. The emphasis of this work is on determining, quantitatively, the differences in the calculated PET image yields resulting from four different sets of input nuclear reaction cross section data.
\end{abstract}

\section{INTRODUCTION}

The goal of radiation therapy is to control the tumor while minimizing the concomitant damage to the surrounding normal tissue. Proton therapy (PT) is increasingly used in clinical radiation therapy because its dose distribution conforms more tightly to the target volume than conventional $x$-ray radiation therapy. The reason is that protons, being charged particles, stop quite sharply at the end of their track, following the much intense ionization density they produce in their Bragg peak immediately before they stop. Figure 1 shows the significant superiority of proton 
therapy compared to that of x-ray radiation therapy. Figure 2 compares the dose distribution of proton and light-ion therapies to the dose distribution of conventional RT. Conventional radiation therapy deposits energy close to the entrance site (after some skin-sparing effect), and also allows the dose deposition to continue beyond the tumor. Since most of the proton's energy is deposited in one area (the so-called spread-out Bragg peak), less energy is deposited into healthy surrounding tissues. The decrease in damage to the normal tissue results in fewer acute and delayed toxicity. As a result, radiation oncologists can increase the radiation dose to the tumor for better therapy [2-4].
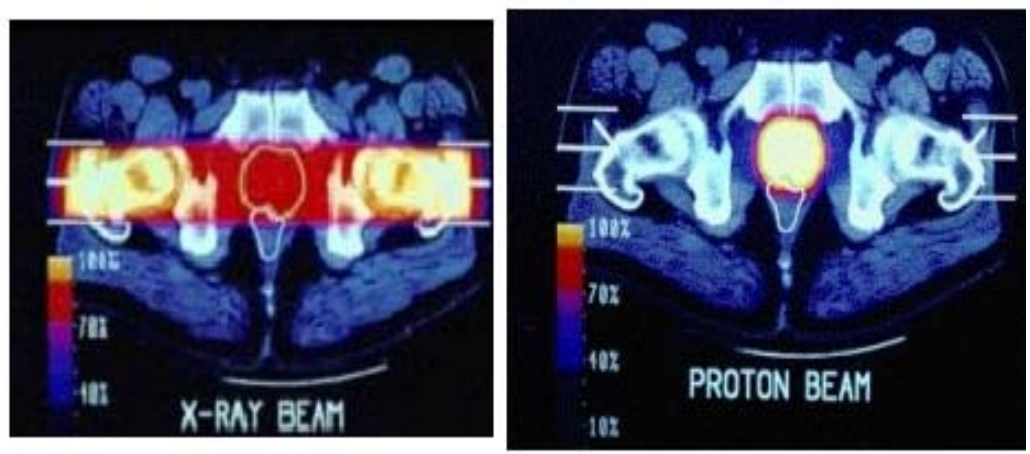

Figure 1. The comparison of energy deposited at a tumor from proton therapy (image on the right) and an x-ray beam radiation (image on the left), with yellow indicating the highest dose [4].

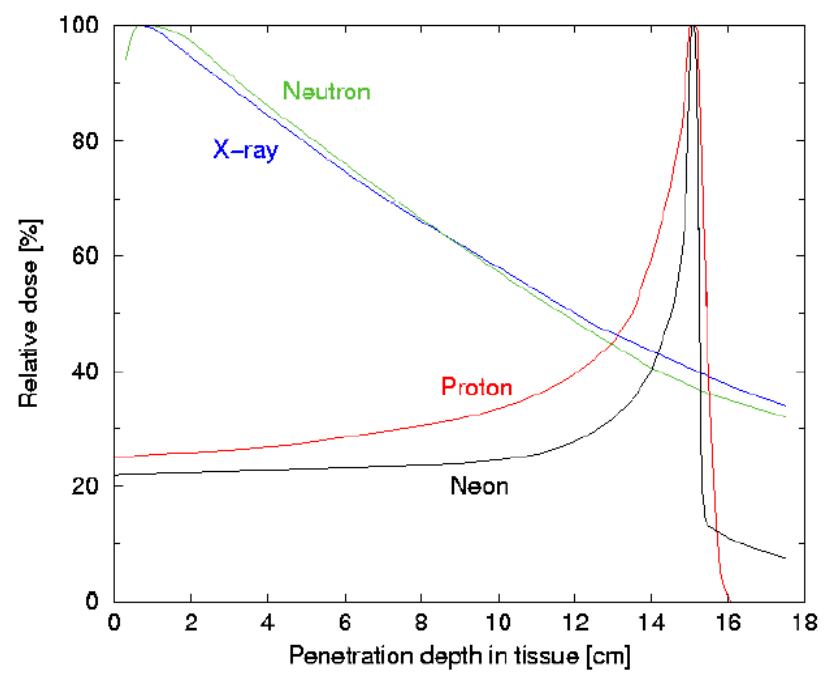

Figure 2. Relative dose distribution of $x$-ray, neutron, proton, and neon radiation therapy. 
One possible way to determine the location of the Bragg peak during or right after a PT session is by positron emission tomography (PET). PET is a medical imaging technique that shows the chemical functioning of organs and tissues, while other imaging, such as X-rays, CT scans, and MRIs, only shows the structure. In general, PET imaging is achieved by introducing positron-emitting radioisotopes into the body. The radioisotopes react with the molecules in the body, and decay to stability by emitting positrons. When a positron encounters an electron in the surrounding tissue, the two particles annihilate turning the mass of the two particles into two $511 \mathrm{keV} \gamma$-rays with opposed momenta. These $\gamma$-rays escape from the human body and can be recorded by external detectors. PET imaging has proven to be very useful in oncology, neurology, cardiology, and psychiatry [5-6].

The previous work at BNL [1] demonstrated that PET is also a potentially powerful tool for quality assurance of PT. One important feature of proton therapy is that it produces small quantities of positron-emitting isotopes along the beam-path through the non-elastic nuclear interaction of protons with target nuclei such as ${ }^{12} \mathrm{C},{ }^{14} \mathrm{~N}$ and ${ }^{16} \mathrm{O}$. These radioisotopes, mainly ${ }^{11} \mathrm{C},{ }^{13} \mathrm{~N}$ and ${ }^{15} \mathrm{O}$, allow PET imaging of the therapy dose. This is especially important when treating inhomogeneous organs such as the lungs or the head-and-neck, where the calculation of the expected dose distribution for treatment planning is more difficult. In this paper, we present an analytical calculation of the yield of positron emitters produced by proton beams up to $250 \mathrm{MeV}$ in conjunction with the Monte Carlo simulation predictions in the previous work [1]. The predictions of the positron emitter distributions are produced through analytical formulas and simple calculations, which can be used as a benchmark for detailed simulation or experiments. It is also demonstrated that the calculated prediction of the positron emitter production distribution is strongly dependent on the nuclear reaction cross section data for a given proton beam flux and target elemental composition. The emphasis of this study is to determine quantitatively the differences between the calculated distributions resulting from four different sets of the nuclear reaction cross section data available. It is important especially in the region near the Bragg peak, which is critical to the success of PET imaging for verification of proton beam location and dosimetry. 


\section{NUCLEAR REACTIONS LEADING TO POSITRON EMITTER PRODUCTIONS}

During PT, many isotopes are produced through different nuclear reactions. When protons are introduced into a human body, they react with ${ }^{16} \mathrm{O},{ }^{14} \mathrm{~N}$, and ${ }^{12} \mathrm{C}$, all which can result in the production of positron-emitting isotopes. The radioisotopes, ${ }^{11} \mathrm{C},{ }^{13} \mathrm{~N}$, and ${ }^{15} \mathrm{O}$, are the three main positron emitters produced. Table 1 summarizes the six main channels that produce these positron-emitting nuclei in the human tissue. The threshold energy is the minimum kinetic energy in the initial state needed for the nuclear reaction to proceed.

\begin{tabular}{|cccc|}
\hline Reaction & $\begin{array}{c}\text { Threshold } \\
\text { Energy } \\
(\mathrm{MeV})\end{array}$ & $\begin{array}{c}\text { Half-life } \\
\text { Time } \\
(\mathrm{min})\end{array}$ & $\begin{array}{c}\text { Positron } \\
\text { Max. Energy } \\
(\mathrm{MeV})\end{array}$ \\
\hline${ }^{12} \mathrm{C}(\mathrm{p}, \mathrm{pn}){ }^{11} \mathrm{C}$ & 20.61 & 20.39 & 0.96 \\
${ }^{16} \mathrm{O}(\mathrm{p}, \mathrm{pn}){ }^{15} \mathrm{O}$ & 16.79 & 2.04 & 1.72 \\
${ }^{16} \mathrm{O}(\mathrm{p}, 2 \mathrm{p} 2 \mathrm{n}){ }^{13} \mathrm{~N}$ & $5.66^{\mathrm{c})}$ & 9.97 & 1.19 \\
${ }^{16} \mathrm{O}(\mathrm{p}, 3 \mathrm{p} 3 \mathrm{n}){ }^{11} \mathrm{C}^{\mathrm{b})}$ & $27.50^{\mathrm{c})}$ & 20.39 & 0.96 \\
${ }^{14} \mathrm{~N}(\mathrm{p}, \mathrm{pn}){ }^{13} \mathrm{~N}$ & 11.44 & 9.97 & 1.19 \\
${ }^{14} \mathrm{~N}(\mathrm{p}, 2 \mathrm{p} 2 \mathrm{n}){ }^{11} \mathrm{C}^{\mathrm{a})}$ & $3.22^{\mathrm{c})}$ & 20.39 & 0.96 \\
\hline
\end{tabular}

a): $(p, 2 p 2 n)$ is inclusive of $(p, \alpha)$

b): $(\mathrm{p}, 3 \mathrm{p} 3 \mathrm{n})$ is inclusive of ( $\mathrm{p}, \alpha \mathrm{pn})$

c): The listed thresholds refer to (p, $\alpha)$ and (p, $\alpha p n)$

Table 1. The six main channels for positron- emitter production in human body [1].

Table 2 summarizes the other 15 more exotic nuclear reactions leading to the production of positron emitters that are not included in this study. Since ${ }^{13} \mathrm{C},{ }^{15} \mathrm{~N}$ and ${ }^{18} \mathrm{O}$ are rarely found in the human body, the reactions induced by protons with these isotopes are not taken into account. The cross sections of the radioactive capture reactions, $(\mathrm{p}, \gamma)$, are typically micro-barns, or three orders of magnitude smaller than the six main channels 
listed in Table 1. Also, due to the very small quantities of production of ${ }^{10} \mathrm{C}$ and ${ }^{14} \mathrm{O}$ isotopes, the uncertainties in their cross section data would make the calculation results meaningless. Therefore, these reactions are excluded in this study. The productions of isotope ${ }^{14} \mathrm{O}$ are also not investigated due to its short half-life time $(1.18 \mathrm{~min})$, even though the production cross sections is not so small $(\sim 100 \mathrm{mb}$ around $10 \mathrm{MeV})$.

\begin{tabular}{|cccc|}
\hline Reaction & $\begin{array}{c}\text { Threshold } \\
\text { Energy }(\mathrm{MeV})\end{array}$ & $\begin{array}{c}\text { Half-life Time } \\
\text { (min) }\end{array}$ & $\begin{array}{c}\text { Positron Max. } \\
\text { Energy (MeV) }\end{array}$ \\
\hline${ }^{12} \mathrm{C}(\mathrm{p}, \mathrm{p} 2 \mathrm{n}){ }^{10} \mathrm{C}$ & 34.5 & 0.32 & 1.87 \\
${ }^{12} \mathrm{C}(\mathrm{p}, \gamma){ }^{13} \mathrm{~N}$ & 0.0 & 9.97 & 1.19 \\
${ }^{13} \mathrm{C}(\mathrm{p}, \mathrm{p} 2 \mathrm{n}){ }^{11} \mathrm{C}$ & 25.5 & 20.30 & 0.96 \\
${ }^{13} \mathrm{C}(\mathrm{p}, \mathrm{n}){ }^{13} \mathrm{~N}$ & 3.2 & 9.97 & 1.19 \\
${ }^{14} \mathrm{~N}(\mathrm{p}, \mathrm{n} \alpha)^{10} \mathrm{C}$ & 17.2 & 0.32 & 1.87 \\
${ }^{14} \mathrm{~N}(\mathrm{p}, \gamma){ }^{15} \mathrm{O}$ & 0.0 & 2.04 & 1.72 \\
${ }^{14} \mathrm{~N}(\mathrm{p}, \mathrm{n}){ }^{14} \mathrm{O}$ & 6.6 & 1.18 & 1.81 \\
${ }^{15} \mathrm{~N}(\mathrm{p}, \mathrm{n} \alpha)^{11} \mathrm{C}$ & 14.7 & 20.30 & 0.96 \\
${ }^{15} \mathrm{~N}(\mathrm{p}, \mathrm{nd})^{13} \mathrm{~N}$ & 20.4 & 9.97 & 1.19 \\
${ }^{15} \mathrm{~N}(\mathrm{p}, \mathrm{t}){ }^{13} \mathrm{~N}$ & 13.8 & 9.97 & 1.19 \\
${ }^{15} \mathrm{~N}(\mathrm{p}, \mathrm{n}){ }^{15} \mathrm{O}$ & 3.8 & 2.04 & 1.72 \\
${ }^{16} \mathrm{O}(\mathrm{p}, \gamma){ }^{17} \mathrm{~F}$ & 0.0 & 1.07 & 1.74 \\
${ }^{16} \mathrm{O}(\mathrm{p}, 3 \mathrm{p} 4 \mathrm{n})^{10} \mathrm{C}$ & 39.1 & 0.32 & 1.87 \\
${ }^{16} \mathrm{O}(\mathrm{p}, \mathrm{p} 2 \mathrm{n}){ }^{14} \mathrm{O}$ & 30.7 & 1.18 & 1.81 \\
${ }^{18} \mathrm{O}(\mathrm{p}, \mathrm{n}){ }^{18} \mathrm{~F}$ & 2.6 & 109.80 & 0.64 \\
\hline
\end{tabular}

Table 2. Uncommon reactions that produce positron-emitting nuclei [7].

The expected number of nuclear reactions is governed by three factors: nuclear cross sections, the number of incoming particles, and the number of target particles. The nuclear cross section $\sigma$ is defined to be the probability $P$ of the interaction for one target nucleus, when subjected to the particle flux $\Phi$ :

$$
\sigma=\frac{P}{\Phi}
$$

In the following sections, we discuss the issues related to proton flux, target particle density, nuclear cross sections, beam depth, positron productions and activities. 


\section{PROTON FLUX AND TARGET DENSITY CALCULATIONS}

The initial proton flux, $\Phi_{0}(\vec{r}, t)$, describes the number of protons that entering a human body per second per unit area perpendicular to the beam direction. Inside of the human body, the proton flux decreases along the beam path primarily due to absorptive non-elastic nuclear interactions, such as those leading to the production of PET isotopes. For a proton beam passing through tissue with an initial energy of $E_{0}=250 \mathrm{MeV}$, approximately $31 \%$ of particles are removed from the beam by nuclear processes before the end of its range [8]. It has been shown that if $P_{a b s}(E)$ is the probability that a particle with energy $E$ will be absorbed and undergo a nuclear reaction before the end of its range, the proton flux $\Phi(z)$ decreases as the depth $z$ increases [9]:

$$
\Phi(z)=\Phi_{0} \cdot\left[\frac{\left(1-P_{a b s}\left(E_{0}\right)\right)}{\left(1-P_{a b s}(E(z))\right)}\right]
$$

During a treatment session at a given depth $z$, the total number of protons $N_{p}$ delivered through an area $A(z)$ perpeticular to the beam direction is related to the total average dose $D(z)$, measures in Grays, through:

$$
D=\frac{10^{11} q N_{p}}{A}\left|\frac{d E}{d \lambda}\right|_{T}
$$

Where $\mathrm{q}$ is the elementary charge in Coulumb, $A$ is the target area in $\mathrm{mm}^{2}$, and $(d E / d \lambda)_{T}$ is the total stopping power of target material. The number of protons used in this study is $2 \times 10^{6}$ with initial energy of $250 \mathrm{MeV}$. This proton beam was estimated to produce an average absorbed dose of 1 Gray in the last $8.5 \mathrm{~cm}$ of its track [1].

The production of positron-emitting nuclei depends on composition of the target. The elemental compositions of human muscle and human fat by percent mass are listed in Table 3. In this study, we use the 4-component elemental composition of soft tissue published by ICRU with $10.11 \%$ hydrogen, $11.11 \%$ carbon, $2.60 \%$ nitrogen, and $76.18 \%$ oxygen [1]. 


\begin{tabular}{|c|cc|}
\hline Element & Human muscle & Human fat \\
\hline Oxygen & 72.9 & 27.8 \\
Hydrogen & 10.2 & 11.4 \\
Carbon & 12.3 & 59.8 \\
Nitrogen & 3.5 & 0.7 \\
\hline
\end{tabular}

Table 3. The elemental compositions of human muscle and human fat by percent mass [7].

\section{NUCLEAR REACTION CROSS SECTIONS}

If the incoming proton beam flux and target density are fixed, the positron emitter production is determined by the cross section data used in the calculations. A nuclear cross section is a physical property of a nuclear reaction, given by nature. Humans can only try to measure cross sections by experiments, then compile the results with existing nuclear reaction models. However, the currently available data are very limited -- either incomplete or un-compiled -- because very few experimental measurements have been performed on these reactions especially in the low energy range below $50 \mathrm{MeV}$. Four sets of nuclear reaction cross section data were used for this study:

1). Data extracted from the emission spectra of recoils in the ENDF electronic file provided by the ICRU Report 63 [10] used by J. Beebe-Wang et al. in reference [1];

2). Data from "Experimental Nuclear Reaction Data File (EXFOR)" maintained by National Nuclear Data Center at BNL [11] used by K. Parodi et al. in reference [12];

3). Data from TERA 95/19 TRA15 [13] used by A. Del Guerra et al. in reference [14];

4). Data from 8 different resources during 1962-1996 collected by D. Litzenberg in his $\mathrm{Ph} . \mathrm{D}$. dissertation [7].

We display and compare the cross section data of the six main channels amongst these four sources in Figures 3-8. In the higher energy range, where some data are not available, extrapolation was used to extend the data to $250 \mathrm{MeV}$. 


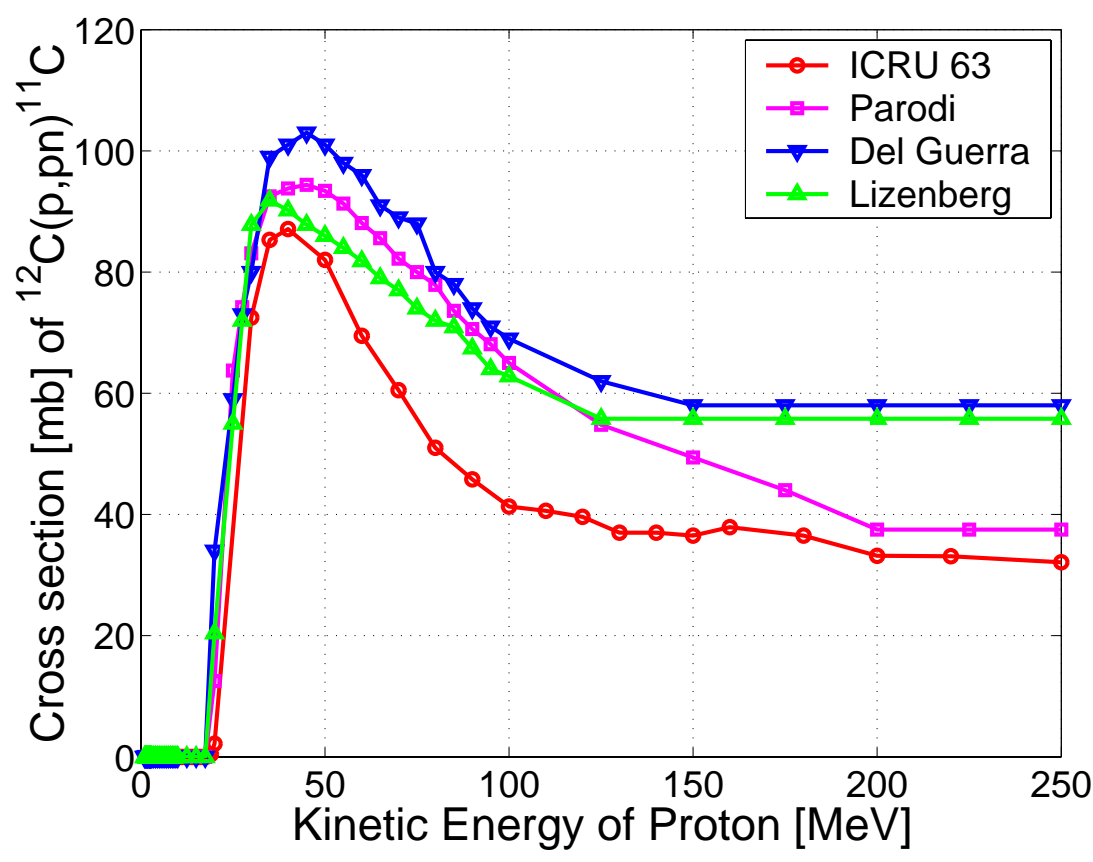

Figure 3. Nuclear reaction cross sections of ${ }^{12} \mathrm{C}(\mathrm{p}, \mathrm{pn})^{11} \mathrm{C}$. The data from four different resources are presented for comparison.

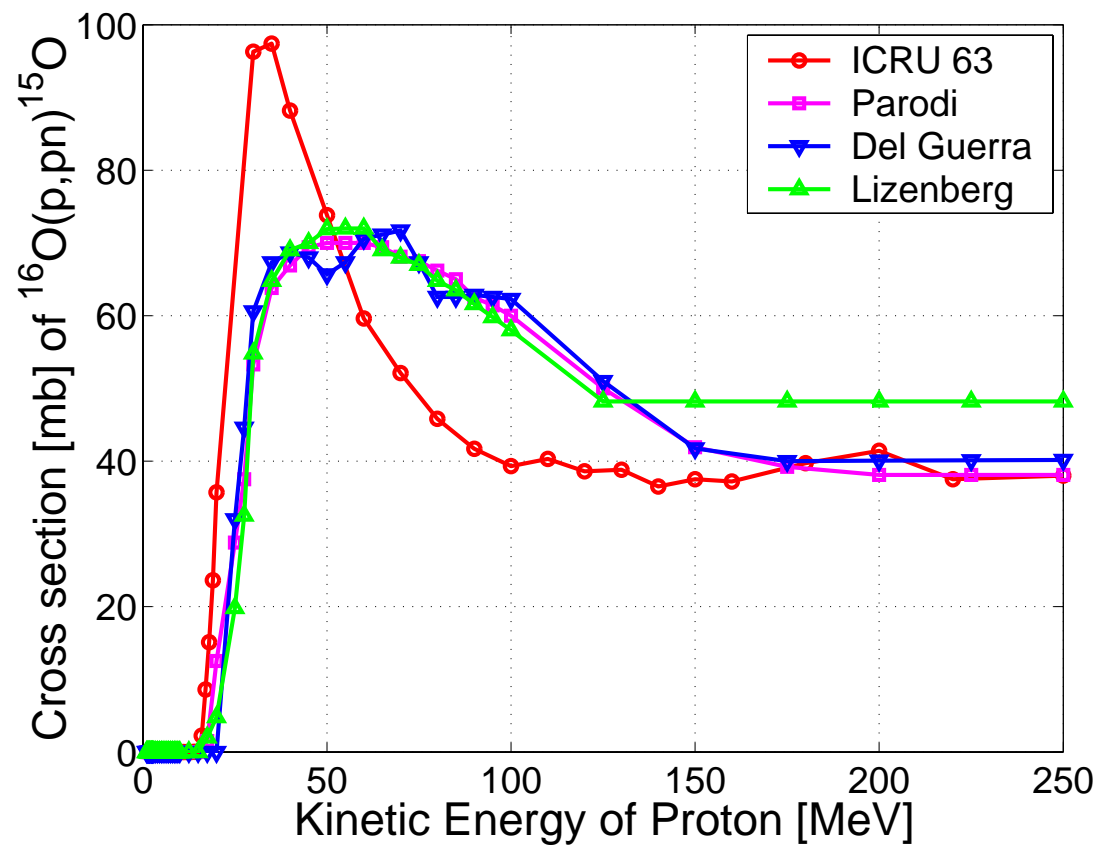

Figure 4. Nuclear reaction cross sections of $16 \mathrm{O}(\mathrm{p}, \mathrm{pn})^{15} \mathrm{O}$. The data from four different resources are presented for comparison. 


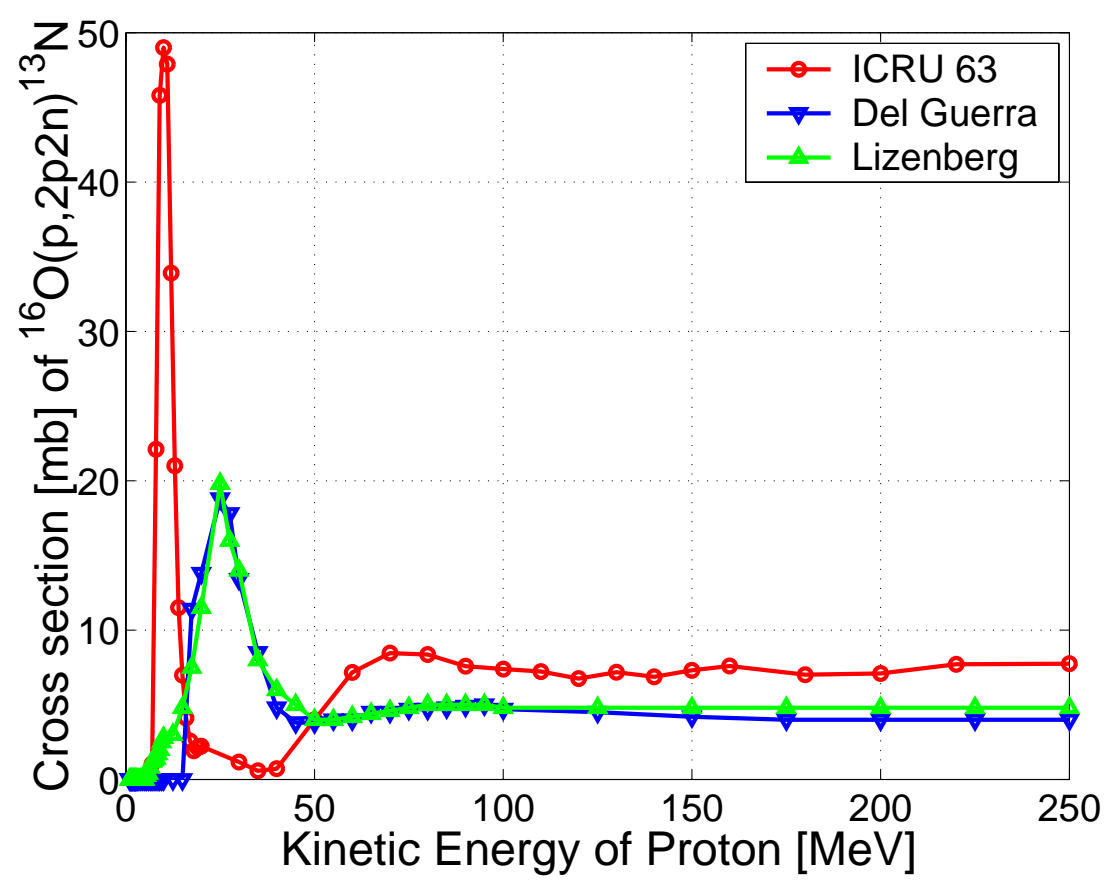

Figure 5. Nuclear reaction cross section of $16 \mathrm{O}(\mathrm{p}, 2 \mathrm{p} 2 \mathrm{n})^{13 \mathrm{~N}}$. The data from three different resources are presented for comparison.

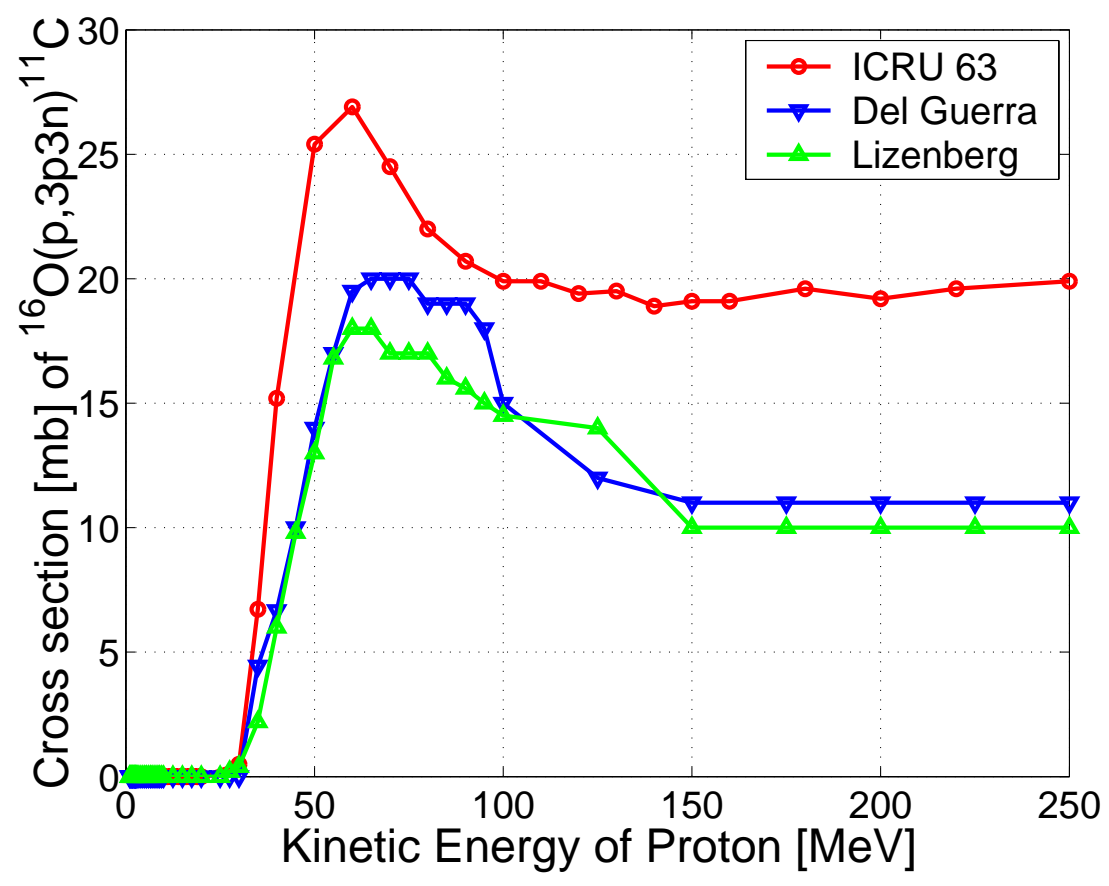

Figure 6. Nuclear reaction cross section of $16 \mathrm{O}(\mathrm{p}, 3 \mathrm{p} 3 \mathrm{n})^{11} \mathrm{C}$. The data from three different resources are presented for comparison. 


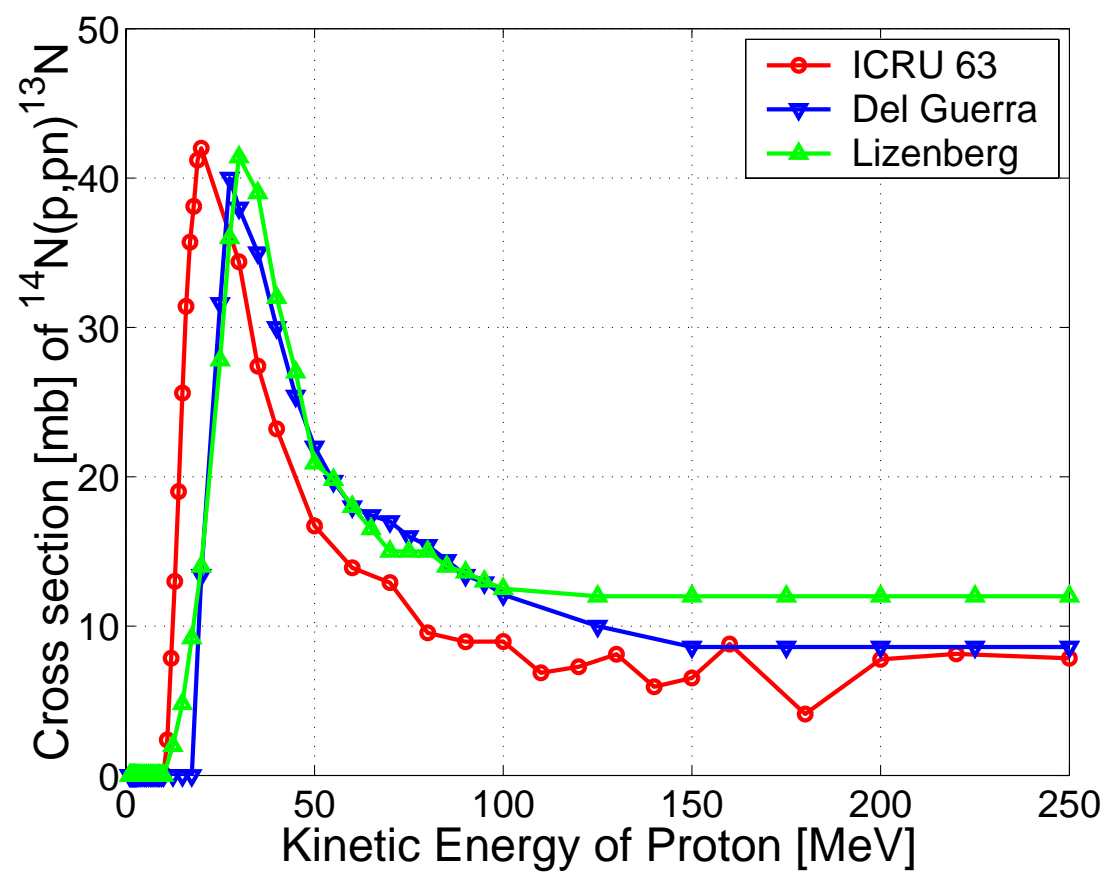

Figure 7. Nuclear reaction cross section of ${ }^{14} \mathrm{~N}(\mathrm{p}, \mathrm{pn})^{13} \mathrm{~N}$. The data from three different resources are presented for comparison.

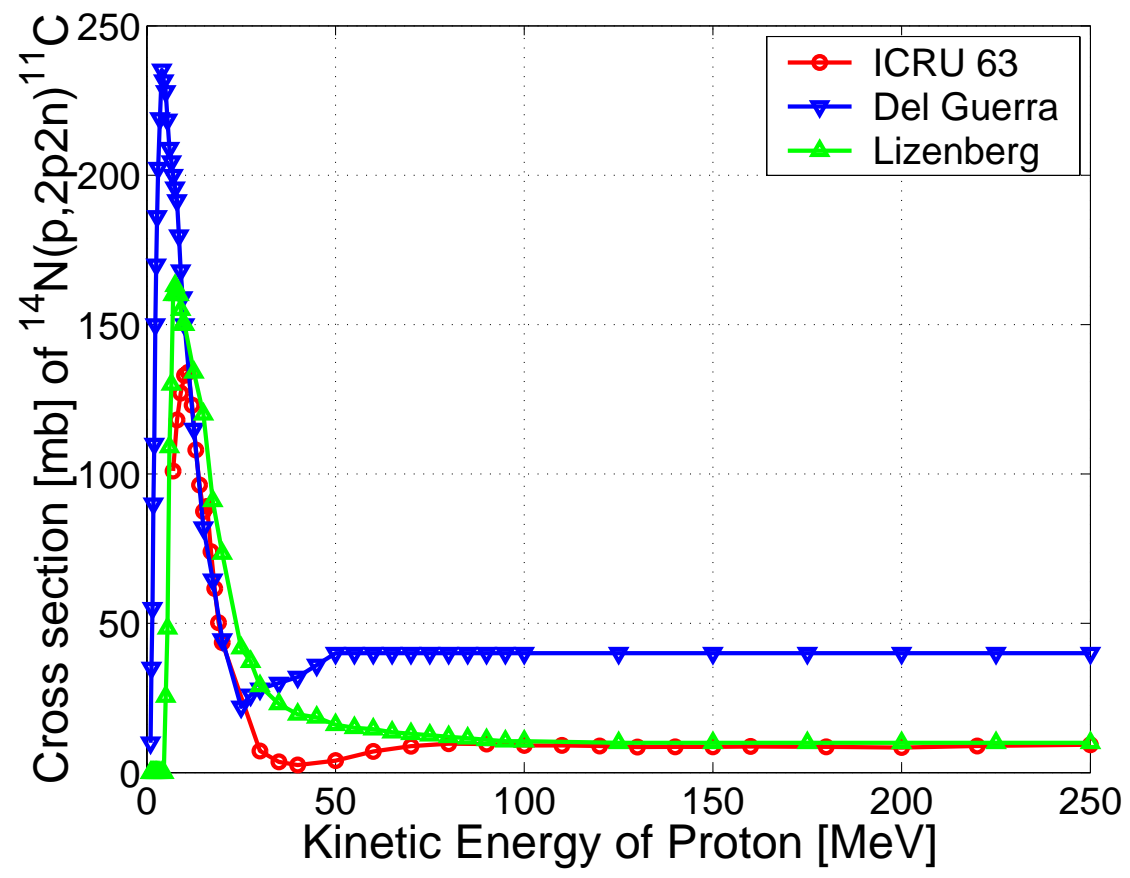

Figure 8. Nuclear reaction cross section of ${ }^{14} \mathrm{~N}(\mathrm{p}, 2 \mathrm{p} 2 \mathrm{n})^{11} \mathrm{C}$. The data from three different resources are presented for comparison. 


\section{STOPPING POWER AND PROTON BEAM DEPTH}

When a proton beam enters the body, it comes in contact with a number of materials, such as bone, muscle, fat, and blood. The material type $i$ has its own stopping power $(d E / d \lambda)_{i}$. This must be taken into account when calculating the total stopping power $(d E / d \lambda)_{T}$ of target material as:

$$
\rho_{T}\left(\frac{d E}{d \lambda}\right)_{T}=\sum_{i} \rho_{i}\left(\frac{d E}{d \lambda}\right)_{i}
$$

where $\rho_{i}$ and $(d E / d \lambda)_{i}$ are the density and the stopping power of each individual element in the target. The values of stopping powers are obtained from experiments and simulations and are similar to cross-sections in the sense that they are natural properties of the materials. Humans cannot change stopping power of human tissue and can only try to measure it as accurately as possible.

In order to express the production of positron emitter as a function of depth $z$ along the beam path instead of a function of proton energy $E$, we need to express the depth $z$ as a function of energy $E$. This is accomplished by numerically integrating the inverse of $\left|\frac{d E}{d z}\right|=\rho_{T}\left|\frac{d E}{d \lambda}\right|_{T}$ as follows:

$$
z(E)=\int_{E_{0}}^{E}\left(\rho_{T}\left|\frac{d E}{d \lambda}\right|_{T}\right)^{-1} d E
$$

In this study, soft tissue (ICRU 4-component) is assumed to be a homogenous material with a 0.55 ratio of the averaged atomic number to atomic mass $(Z / A)$, and a density of $1.0 \mathrm{~g} / \mathrm{cm}^{3}$. The total stopping power $(d E / d \lambda)_{T}$ as function of the proton energy $E$ is obtained from the PSTAR database maintained by Physics Laboratory, NIST [15]. 


\section{PRODUCTION AND ACTIVITY CALCULATIONS}

Inside of any small treatment volume $A d z$ located at $\vec{r}$ and at time $t$ during the PT, the number $N_{i p}$ of positron emitter type $i$ produced by nuclear reaction process $p$ changes with a rate $d N_{i p}(\vec{r}, t) / d t$ governed by the rate of production and decay:

$$
\frac{d N_{i p}(\vec{r}, t)}{d t}=\sigma_{i p}(E(\vec{r})) \Phi(\vec{r}, t) n_{i}(\vec{r}) A d z-\lambda_{i} N_{i p}(\vec{r}, t)
$$

The first term on the right side of the equation (6.1) represents the production rate. It is propotionoal to the nuclear cross section $\sigma_{i p}(E(\vec{r}))$ leading to production of positronemitting nuclei of type $i$ through channel $p$, the proton beam flux $\Phi(\vec{r}, t)$, and the target density $n_{i}(\vec{r})$ in the small volume $A d z$. Here $A$ is the transverse beam size, and $d z$ is the increment of the depth.

The second term on the right side of the equation (6.1) represents the decay rate. Here $\lambda_{i}$ is the decay constant of isotope type $i$, defined as the instant rate of radioactive decay:

$$
N_{i}(\vec{r}, t)=N_{i}(\vec{r}, 0) e^{-\lambda_{i} t}
$$

and $N_{i}(\vec{r}, 0)$ and $N_{i}(\vec{r}, t)$ are the number of positron-emitting nuclei of type $i$ at time zero and time $t$ during a decay process, respectively.

Proton beam flux may be either continuous or pulsed over the treatment time. Dealing with continuous beams is less complicated than dealing with pulsed beams. For a continuous beam, or a beam with constant flux over one beam-pulse duration $T_{o n}$, we can obtain $N_{i p}(\vec{r}, t)$ by integral equation (6.1) with initial conditions:

$$
N_{i p}(\vec{r}, t)=\frac{\sigma_{i p}(E(\vec{r})) \cdot \Phi(\vec{r}) \cdot n_{i}(\vec{r}) A d z\left(1-e^{-\lambda_{i} t}\right)}{\lambda_{i}} \quad \text { for } t \leq T_{o n}
$$

and

$$
N_{i p}(\vec{r}, t)=\frac{\sigma_{i p}(E(\vec{r})) \Phi(\vec{r}) n_{i}(\vec{r}) A d z\left(1-e^{-\lambda_{i} T_{o n}}\right)\left(e^{-\lambda_{i}\left(t-T_{o n}\right)}\right)}{\lambda_{i}} \quad \text { for } t \geq T_{o n}
$$


Whether the beam is continuous or pulsed, to find the total production of positron emitting nuclei would one have to sum over all channels and all isotopes:

$$
N(\vec{r}, t)=\sum_{i} \sum_{p} N_{i p}(\vec{r}, t)
$$

In this study, only the production of position emitter is calculated. The decay process will be studied separately with PET imaging simulation. In figures 9-11 we are comparing the results of production ${ }^{15} \mathrm{O},{ }^{13} \mathrm{~N}$, and ${ }^{11} \mathrm{C}$ respectively, amongst four different data resources of nuclear reaction cross sections listed in Section 4.

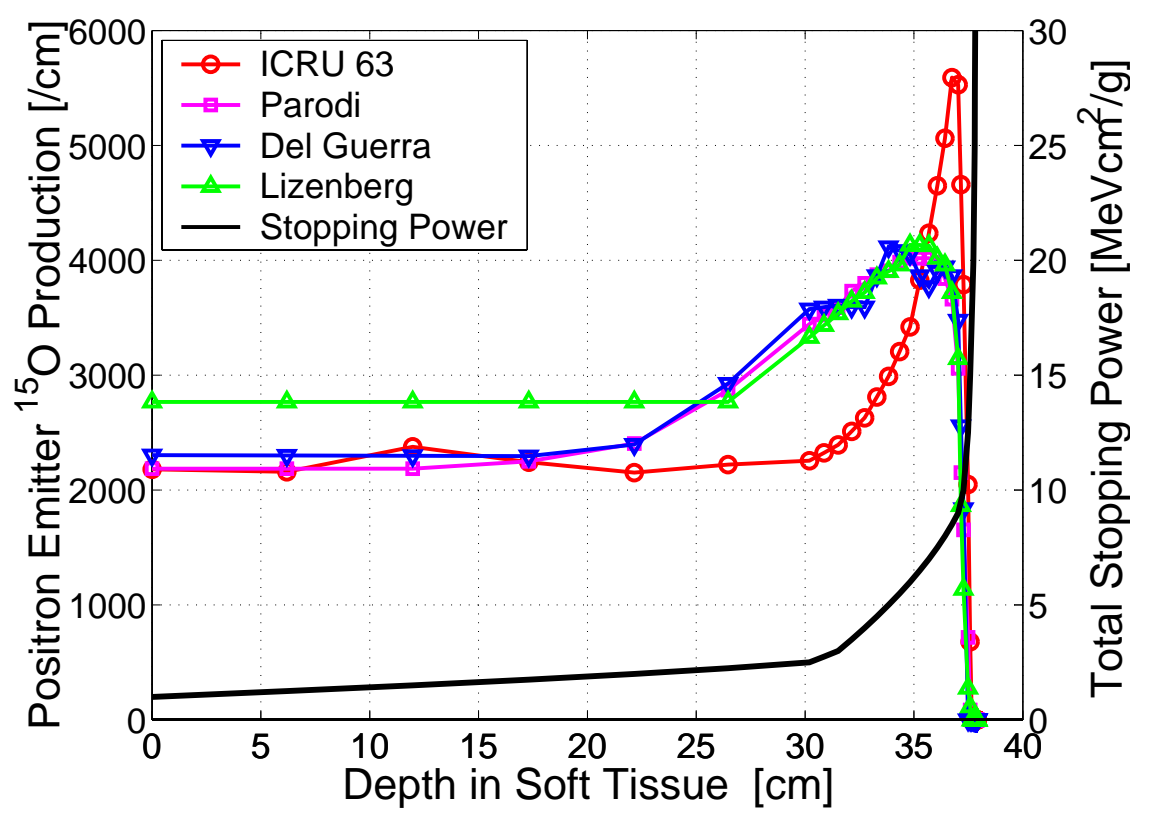

Figure 9. The estimated production of $\mathrm{O}^{15}$ isotopes during a proton $\mathrm{RT}$ session. The distributions calculated with four different cross section data resources are presented for comparison. 


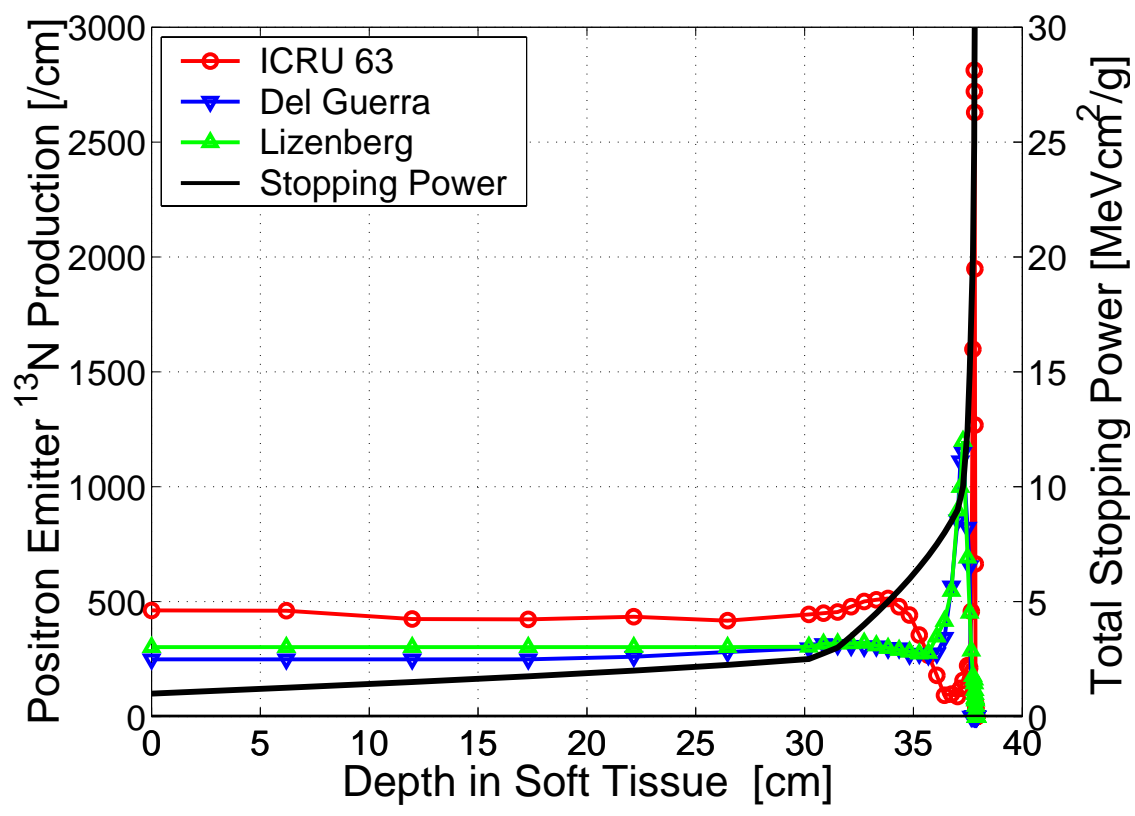

Figure 10. The estimated production of $\mathrm{N}^{13}$ isotopes during a proton RT session. The distributions calculated with three different cross section data resources are presented for comparison.

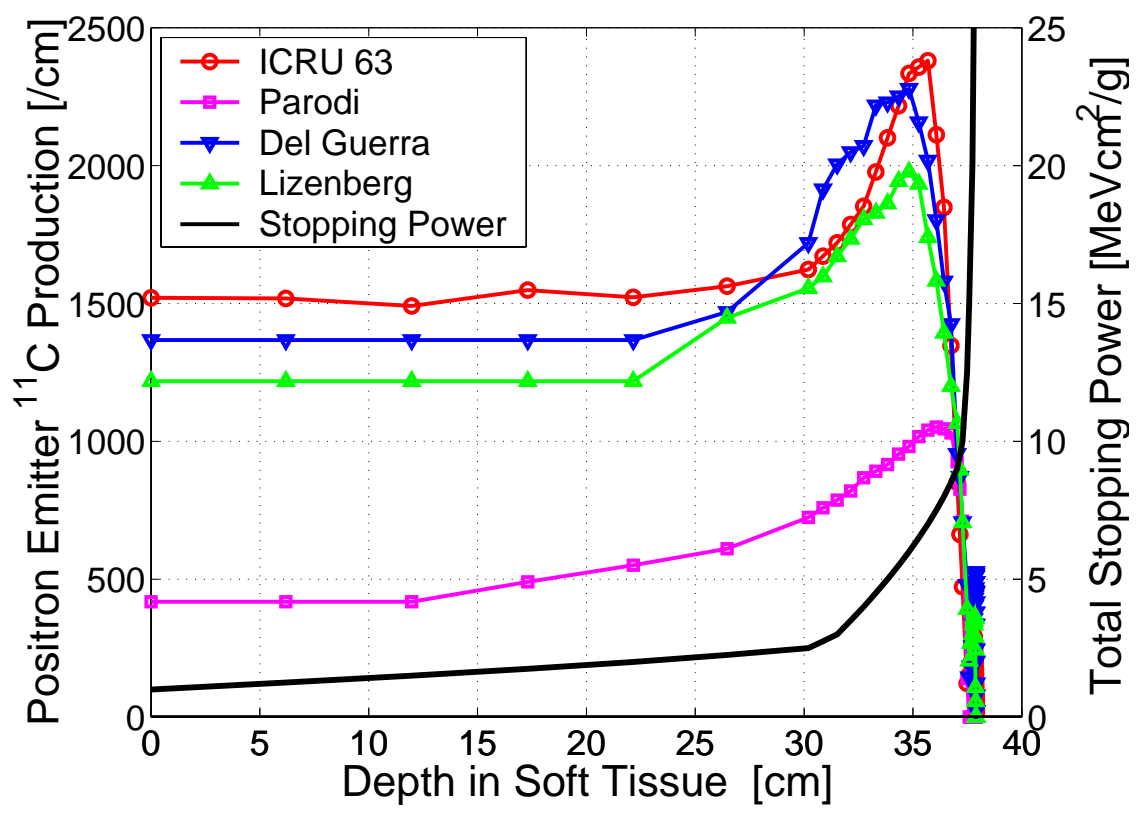

Figure 11. The estimated production of $\mathrm{C}^{11}$ isotopes during a proton RT session. The distributions calculated with four different cross section data resources are presented for comparison. 


\section{DISCUSSIONS AND CONCLUSIONS}

The positron emitter production distributions obtained from this study are in good agreement with the Monte Carlo simulation predictions of our previous work [1]. It supports the conclusion made previously [1] that, with $250 \mathrm{MeV}$ protons and a typical radiotherapy dose to the target volume during a therapy session, PET imaging for quality assurance of PT should be feasible.

The differences in positron emitter production distributions due to the different resources of nuclear reaction cross section data are easily observed in Figures 9-11. In the depth range between $0 \mathrm{~cm}$ and $30 \mathrm{~cm}$ the production of the isotopes is almost without structure, reflecting the fact that the cross sections remain almost unchanged in the energy range above $100 \mathrm{MeV}$. In this range, the yields calculated with four different cross section data resources reach fairly good agreement with each other, except the ${ }^{11} \mathrm{C}$ isotope production obtained with the data from reference [11]. The $\mathrm{C}^{11}$ isotope production calculated with the data from reference [11] used by Parodi et al is only about $30 \%$ of production compared with the other data resources, mainly because only one of the three major channels producing ${ }^{11} \mathrm{C}$ isotope is taken into account [12]. In the depth range between $30 \mathrm{~cm}$ and $38 \mathrm{~cm}$ where the Bragg peak is located, the calculated productions with data resources are significantly different for all three isotopes (see Figures 9-11). The high yield of ${ }^{13} \mathrm{~N}$ and ${ }^{15} \mathrm{O}$ calculated with data ICRU 63 report is credited to the cross section data of reactions $16 \mathrm{O}(\mathrm{p}, 2 \mathrm{p} 2 \mathrm{n})^{13 \mathrm{~N}}$ and $16 \mathrm{O}(\mathrm{p}, \mathrm{pn})^{15 \mathrm{O}}$ in the low energy range which only became available during the year 2000 [10].

This investigation shows that there is an on going need to develop a library of accurate cross section data for proton and neutron-induced reactions on the elements in human tissue. A reliable simulation or calculation depends upon accurate cross section data, especially for the investigations in the region near the Bragg peak where accurate 
cross section data in the low energy range (below $50 \mathrm{MeV}$ ) are needed. This is critical to the success of PET imaging for verification of the Bragg peak location of the proton beam, and for dosimetry.

This study only includes fundamental physical processes leading to positron emitter production. Since many detailed physical processes are not included in this study, more extensive investigations are necessary. However, the mathematical formulations and the simple programs developed for this study can provide a useful tool for the estimate of expected results in the future work. The results from this study can be used as a benchmark for detailed simulation or experiments in the future.

\section{ACKNOWLEDGMENT}

The authors would like to thank Drs. P. Vaska, F. A. Dilmanian, D. J. Schlyer and A. G. Ruggiero for their discussions and suggestions on the subject.

\section{REFERENCES}

[1] J. Beebe-Wang, F.A. Dilmanian, S. Peggs, D.J. Schlyer, P. Vaska " Feasilility of Positron Emission Tomography of Dose Distribution in Proton Beam Cancer Therapy", Proccedings of EPAC 2002, p.2721-2723 (2002).

[2] "Proton Beam Therapy at Loma Linda University Medical Center." Loma Linda University. http://www.llu.edu/proton/index.html

[3] Daniel Miller, "A review of proton beam radiation therapy." Medical Physics, 22 (11), (November 1995) p.1943.

[4] "How Proton Therapy Works." The National Association for Proton Therapy. http://www.proton-therapy.org/howit.htm.

[5] "Positron Emission Tomography." Biomedical Research Foundation of Northwest Louisiana. http://www.biomed.org/pet.html.

[6] "Positron Emission Tomography." Austin and Repatriation Medical Centre. http://www.austin.unimelb.edu.au/dept/nmpet/pet/index.html 
[7] Dale Litzenberg. "On-line Monitoring and PET Imaging of the Positron-Emitting Activity Created in Tissue by Proton Radiotherapy Beams." Ph.D. Dissertation, Department of Physics, University of Michigan (1997).

[8] J.F. Janni, "Proton Range- Energy Tables $1 \mathrm{KeV}-10 \mathrm{GeV}$, Atomic Data and Nuclear Data Tables,vol. 27, no. 2/3. (March/May 1982).

[9] U.Oelfke, G. Lam, and M. Atkins, "Proton dose monitoring with PET: quantitative studies in Lucite" Physics in Medicine and Biology, 41, (1996) p. 177-196.

[10] ICRU Report 63, "Nuclear Data for Neutron and Proton Radiotherapy and for Radiation”, MD, USA (2000).

[11] “Experimental Nuclear Reaction Data File.” Energy Sciences and Technology Department , Brookhaven National Laboratory. http://www.nndc.bnl.gov/nndc/exfor/

[12] Parodi, K., Enghardt, W. and Haberer, T., "In-beam PET measurements of $\beta^{+}$ radioactivity induced by proton beams." Physics in Medicine and Biology, 47, (2002) 21.

[13] D. Mukhopadhyay, A. Del Guerra, and O. Di Domenico, "For PET- monitored hadrontherapy, TERA 95/19 TRA15.

[14] Del Guerra, A. and Di Domenico, G., "Positron Emission Tomography as an aid to in 'vivo' dosimetry for proton radiotherapy: a Monte Carlo simulation," TERA 93/10 TRA 9, August 1993.

[15] "Stopping-Power and Range Tables for Electrons, Protons, and Helium Ions, Physical Reference Data", Physics Laboratory, NIST, 2003. http://physics.nist.gov/PhysRefData/Star/Text/PSTAR.html 